\title{
Plantar Exostosis in a Malunited Calcaneal Fracture: A rare complication
}

\author{
by Asif Sultan ${ }^{1 \rrbracket}$, Tahir Ahmad Dar ${ }^{1}$, Mohd Iqbal Wani ${ }^{1}$, Mubashir Maqbool Wani ${ }^{1}$, Samina Shafi ${ }^{2}$
}

The Foot and Ankle Online Journal 3 (11): 2

Calcaneal fractures are very common and are associated with many complications irrespective of the treatment method chosen. We present a case of malunited calcaneal fracture with a rare complication of an exostosis in the middle $3^{\text {rd }}$ of the plantar surface of calcaneus which clinically produced a large bony swelling on the sole of the foot causing pain and abnormal gait. This type of plantar calcaneal exostosis to our knowledge has not been previously reported. The patient was managed operatively by exostectomy with subtalar arthrodesis and had a good result at 2 years follow-up.

Key words: Calcaneus, fracture, complications, plantar exostosis, exostectomy, subtalar arthrodesis.

This is an Open Access article distributed under the terms of the Creative Commons Attribution License. It permits unrestricted use, distribution, and reproduction in any medium, provided the original work is properly cited. @The Foot and Ankle Online Journal (www.faoj.org)

$\mathrm{T}$ he calcaneus is the most frequently fractured tarsal bone, with calcaneal fractures accounting for $65 \%$ of all tarsal injuries and approximately $2 \%$ of all fractures. ${ }^{1}$ Most of these fractures occur in male industrial workers and lead to significant economic impact because of long-term disability. ${ }^{1}$ Even though recent studies have shown better results with surgical treatment ${ }^{2-5}$ and less invasive stabilization systems ${ }^{3-5}$, the treatment of calcaneal fractures remains controversial because of the suboptimal results of treatment and the incidence of complications associated with both conservative and surgical methods. ${ }^{6-9}$

Address correspondence to: Asif Sultan, Government Hospital for Bone and Joint Surgery. Barzullah, Srinagar, India 190005.

Email-drasifsultan@yahoo.com

\footnotetext{
${ }^{1}$ Government Hospital for Bone and Joint Surgery, Barzullah. Srinagar, India 190005.

${ }^{2}$ Resident Government Medical College, Srinagar, India 190005.
}

On reviewing the literature for the treatment of symptomatic, malunited calcaneal fractures, the general consensus suggests that resection of impinging bone with isolated subtalar arthrodesis for subtalar joint arthritis yields the best long term results. ${ }^{10-23}$ Moreover, some surgeons also do endoscopic calcaneal ostectomies for calcaneofibular impingement with good results. ${ }^{15}$

We present a case of mismanaged, malunited calcaneal fracture in a young male laborer who had an unusual and rare complication of large exostosis on plantar surface of the middle portion of the calcaneus, which to our knowledge has not been reported. The patient presented with bony swelling on the sole of the foot with a painful limp and was managed operatively by excision of the exostosis with subtalar arthrodesis. The patient was symptom free after a 2 year follow-up. 


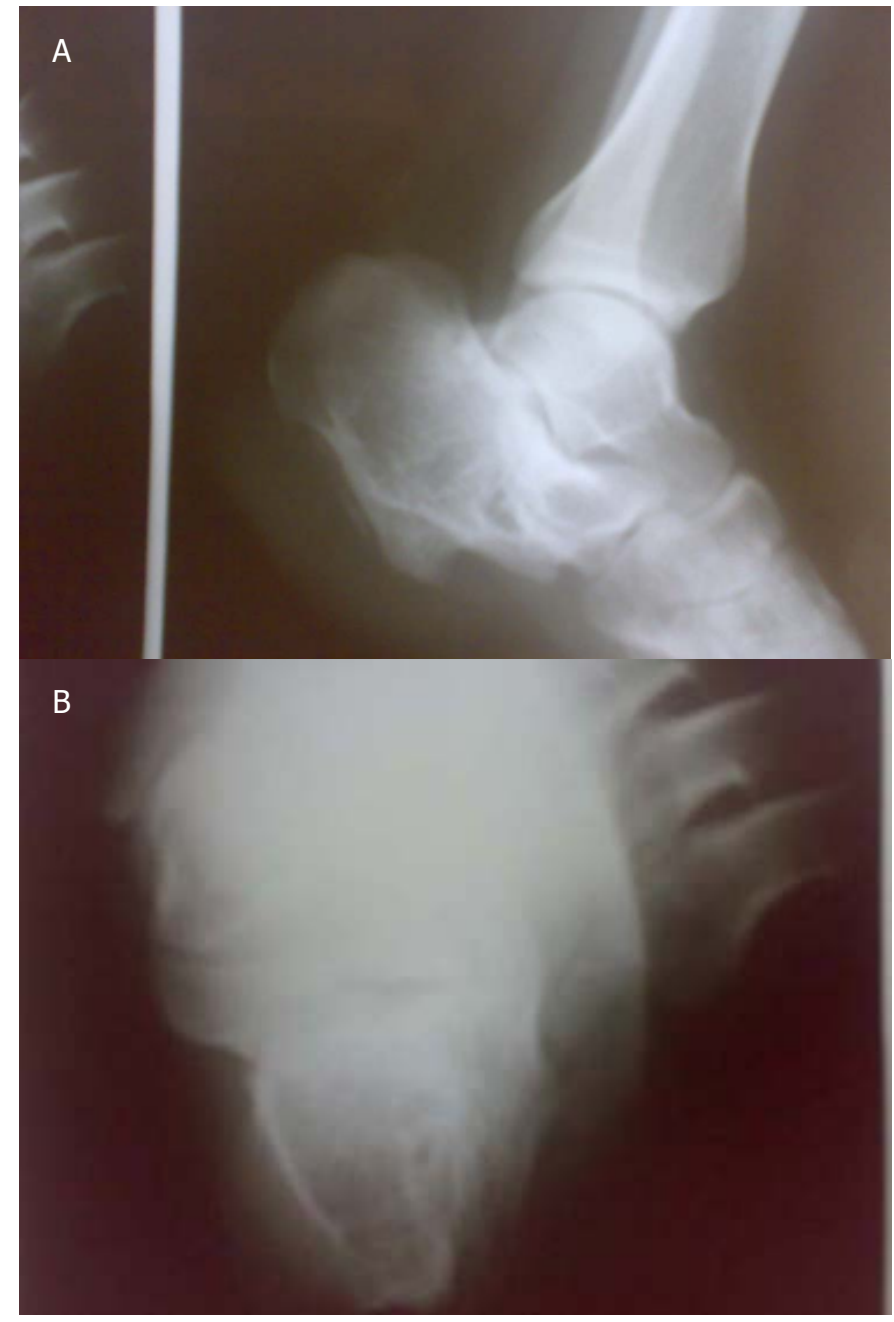

Figure 1A and 1B Lateral (A) and axial (B) view radiographs showing large plantar exostosis, loss of Bohler's angle and subtalar arthritis with normal tibiotalar alignment.

\section{Case report}

A 19 year-old male, manual laborer, living in tribal hilly area presented to us with pain in left rearfoot with prominent swelling on the plantar surface and anterior part of the heel. He had difficulty in walking for the last year. He had suffered a crushing injury to his left foot while at work in his native place when a heavy stone fell on his left foot from the anterolateral aspect.
He had sustained a crushed wound over the left foot which was accompanied by severe pain and the inability to walk. He was managed locally, only for wound care and had no proper medical or orthopedic consultation. This injury healed in 4 weeks, but he still was unable to walk for another two months. After three months of sustained injury, the patient began to walk with a limp and also had noticed hard swelling under the heel. He had always had difficulty in walking afterwards due to pain in the hind foot on weight bearing and abnormal prominence under the heel.

On clinical examination the patient's left foot had an irregular scar of $4 \mathrm{~cm}$ over the anterolateral aspect of the foot. Range of motion at the subtalar joint was restricted and painful with increased heel width and no varus or valgus deformity of heel. There was a prominent bony projection on the plantar surface over the anterior aspect of heel which was tender to touch, with thick, firm, and hypertrophic overlying skin. This region of skin was having major contact with the ground during stance phase, and was associated with an antalgic gait style. Radiographic evaluation showed malunited calcaneal fracture with reduced Bohler's angle, subtalar arthritis, minimally increased heel width and a large exostosis on the plantar surface of calcaneus. (Fig.1A and 1B) The patient was managed operatively under spinal anesthesia. A lateral approach with sharp dissection to raise a full thickness flap from skin to periosteum was performed. A large plantar exostosis was excised along with associated surrounding bursa. There was no cartilaginous cap on the exostosis. Subtalar arthrodesis was performed after denuding cartilage from both the articular surfaces of the calcaneus and talus. The exostosis was used as graft material and fixed with two staples and a short leg cast was applied after closure. (Fig. 2) Sutures were removed after 3 weeks and a short leg cast was reapplied for 3 months with non-weight bearing for 6 weeks and partial weight bearing for another 6 weeks. There were no post operative complications. At 2 years follow-up the patient was pain free with no limp and had a good subtalar fusion. (Fig.3) The patient had returned to his pre-injury employment as manual laborer and the result was deemed good. 


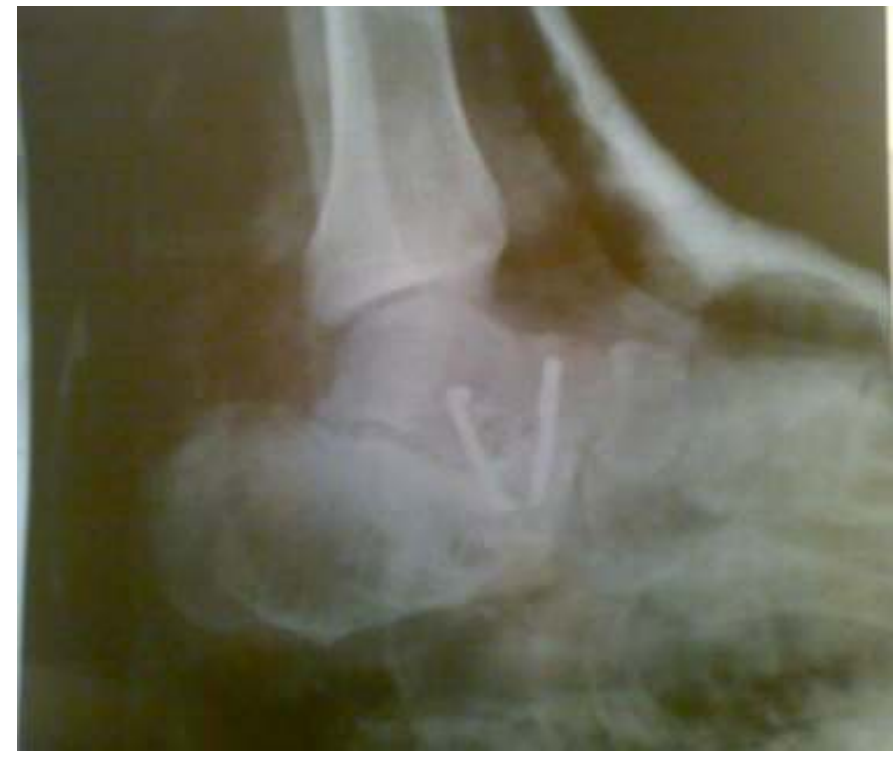

Figure 2 Immediate post operative lateral radiograph after removal of exostosis and subtalar arthrodesis.

\section{Discussion}

Complications of calcaneal fractures occur in the acute and late stages and after operative or nonoperative treatment. ${ }^{1,2-6-9,17,18}$ Acute complications include swelling, fracture blisters, and compartment syndromes. Late complications include arthritis, malunion, lateral exostosis, calcaneofibular abutment, tendon impingement, heel pad problems and reflex sympathetic dystrophy. Complications from nonoperative treatment include arthritis, pain, malunion, and stiffness. Complications associated with operative treatment include wound dehiscence, infection, arthritis, stiffness, and iatrogenic nerve and tendon injury. ${ }^{2}$ Heel pad pain is the second most common site of pain after pain over lateral aspect of heel after a calcaneal fracture ${ }^{2}$ and have thinning with increased mobility of the pad, and a softer, less firm heel pad compared to the normal side. This pain has been attributed to crushing of the heel pad during injury. This was not the cause of pain in our patient as his heel pad was more firm, harder, thickened, and less mobile than the normal side.

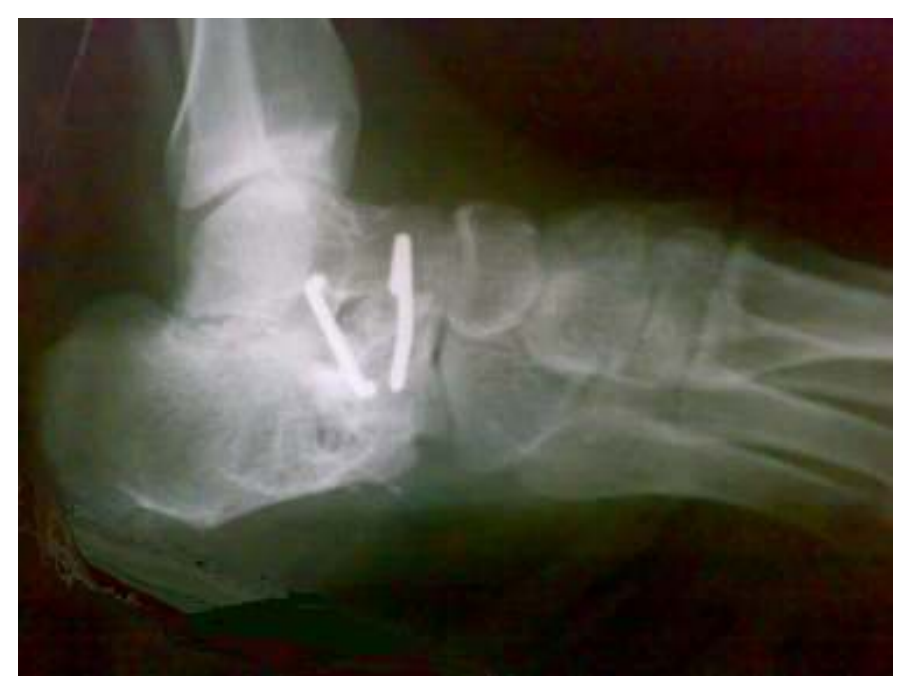

Figure 3 Lateral radiograph at 2 years follow-up showing subtalar fusion with staples in-situ.

Lateral exostosis usually develops after malunion of calcaneal fractures and is a very common complication. Heel exostosis (bony calcaneal spurs) sometimes develop after injury and may also cause chronic heel pain. These develop from the undersurface of the calcaneus in patients with injuries to the plantar cortex. These are as a consequence of proliferative bony changes at the origin of planter fascia. In our patient, the plantar exostosis was on the plantar surface of calcaneus in the middle third of the calcaneus, well anterior to the origin of plantar fascia, thus being a rare inferior surface exostosis rather than heel exostosis. We hypothesize that severe crushing force anteriorly and laterally caused collapse of the calcaneal body to the middle, also fracturing the inferior cortex and taking a large bony fragment plantarly which developed into large bony exostosis.

Post-traumatic arthritis after intra-articular calcaneal fractures may affect the subtalar or calcaneocuboid joint. It may develop either due to articular surface depression or may occur due to secondary cartilage damage from the initial trauma. Subtalar joint arthritis causes pain on weight bearing, aggravated by varus or valgus stressing of the subtalar joint without significant tenderness to the lateral aspect of heel with or without radiological evidence. ${ }^{2}$ 
In our patient, operative intervention was performed using a lateral approach as both inferior wall exostectomy (resection of exostosis) ${ }^{10}$ and isolated subtalar arthrodesis ${ }^{11-13}$ were performed using a single incision. This was indicated for painful subtalar arthritis with normal heel height and normal tibiotalar alignment. (Fig.1) Our result was good with pain free arthrodesis of the subtalar joint. ${ }^{23}$ This procedure of isolated subtalar arthrodesis has been well documented with good results, ${ }^{11-14,23}$ and is consistent with our case.

\section{Conflict of interest}

There is no conflict of interest related to this article

\section{Consent}

Written informed consent was obtained from the patient's guardian for publication of this case report and accompanying images. A copy of the written consent is available for review by the Editor-in-Chief of this journal.

\section{References}

1. Sanders R. Current concepts review: Displaced intraarticular fractures of the calcaneus. JBJS 2000 82A: 225-250.

2. Makki D, Alnajjar HM, Walkay S, Ramkumar U, Watson AJ, Allen PW. Osteosynthesis of displaced intra-articular fractures of the calcaneum: a long-term review of 47 cases. JBJS 2010 92B(5): 693-700.

3. Hernanz González Y, Díaz Martín A, Jara Sánchez F, Resines Erasun C. Early results with the new internal fixator systems LCP and LISS: a prospective study. Acta Orthop Belg 2007 73(1): 60-69.

4. DeWall M, Henderson CE, McKinley TO, Phelps T, Dolan L, Marsh JL. Percutaneous reduction and fixation of displaced intra-articular calcaneus fractures. J Orthop Trauma 2010 24(8): 466-472.

5. Shan SL, Xu JL, Yao SZ, Yu GS, Liu YQ. Minimally invasive plate internal fixation for calcaneal fractures. Chin J Traumatol 2010 13(5): 313-315.
6. Lim EVA, Leung JPF. Complications of intraarticular calcaneal fractures. Clin Ortho Related Research 2001 391:7-16. 7. Walter JH Jr, Rockett MS, Goss LR. Complications of intraarticular fractures of the calcaneus. JAPMA 2004 94(4): 382-388. 8. Reddy V, Fukuda T, Ptaszek AJ. Calcaneus malunion and nonunion. Foot Ankle Clin 2007 12(1): 125-135.

9. Clare MP, Lee WE $3^{\text {rd }}$, Sanders RW. Intermediate to longterm results of a treatment protocol for calcaneal fracture malunions. JBJS 2005 87A (5): 963-973.

10. Abend L, Berstein DA, Wagreich C. Post-traumatic heel deformity. J Foot Surg 1986 25(2): 146-148.

11. Myerson MS, Quill GE Jr. Late complications of fractures of the calcaneus. JBJS 1993 75A: 331-341.

12. Easley ME, Trnka HJ, Schon LC, Myerson MS. Isolated subtalar arthrodesis. JBJS 2000 82A (5): 613-624.

13. Radnay CS, Clare MP, Sanders RW. Subtalar fusion after displaced intra-articular calcaneal fractures: Does initial operative treatment matter? JBJS 2010 92A: 32-43.

14. Sanders R, Fortin PT, Walling, AK. Subtalar arthrodesis following calcaneal fracture. Orthop Trans 1991 15: 656.

15. Lui TH. Endoscopic lateral calcaneal ostectomy for calcaneofibular impingement. Arch Orthop Trauma Surg 2007 127(4): 265-267.

16. Stapleton JJ, Belczyk R, Zgonis T. Surgical treatment of calcaneal fracture malunions and posttraumatic deformities. Clin Podiatr Med Surg 2009 26(1):79-90.

17. Manasseh N, Cherian VM, Abel L. Malunited calcaneal fracture fragments causing tarsal tunnel syndrome: a rare cause. Foot Ankle Surg 2009 15(4): 207-209.

18. Lui TH. Posterior ankle impingement syndrome caused by malunion of joint depressed type calcaneal fracture. Knee Surg Sports Traumatol Arthrosc 2008 16(7): 687-689.

19. Robinson JF, Murphy GA. Arthrodesis as salvage for calcaneal malunions. Foot Ankle Clin 2002 7(1): 107-120.

20. Huang PJ, Fu YC, Cheng YM, Lin SY. Subtalar arthrodesis for late sequelae of calcaneal fractures: fusion in situ versus fusion with sliding corrective osteotomy. Foot Ankle Int 1999 20(3): 166-170.

21. Romash MM. Reconstructive osteotomy of the calcaneus with subtalar arthrodesis for malunited calcaneal fractures. Clin Orthop Relat Res 1993 (290): 157-167.

22. Chen YJ, Huang TJ, Hsu KY, Hsu RW, Chen CW. Subtalar distractional realignment arthrodesis with wedge bone grafting and lateral decompression for calcaneal malunion. J Trauma 1998 45(4):729-737.

23. Rammelt S, Grass R, Zawadski T, Biewener A, Zwipp H. Foot function after subtalar distraction bone-block arthrodesis. A prospective study. JBJS 2004 86B(5): 659-668. 